\title{
Refractory peritonitis by spontaneous perforation of the common bile duct in a patient receiving peritoneal dialysis
}

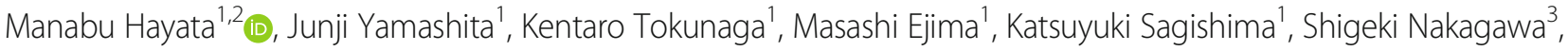 \\ Daisuke Hashimoto ${ }^{3}$, Hideo Baba ${ }^{3}$, Kosuke Maruyama², Yu Kohrogi², Teruhiko Mizumoto², Masashi Mukoyama² \\ and Hidenobu Kamohara ${ }^{1 *}$
}

\begin{abstract}
Background: Patients with end-stage renal disease are in an immune-compromised state, with infection being the most common cause of mortality among those undergoing dialysis.

Case presentation: A 73-year-old woman who had received 6 months of peritoneal dialysis (PD) presented with peritoneal irritation symptoms during hospitalization for examination of PD effluent turbidity. Emergent laparotomy was performed for acute biliary peritonitis, but the cause was undetermined because of severe adhesion, and surgery ended with washing and palliative drainage. Postoperative endoscopic retrograde cholangiopancreatography revealed spontaneous perforation of the common bile duct (CBD), and she was transferred to the intensive care unit (ICU) for treatment of septic shock. Treatment included antibiotics, drainage, mechanical ventilation, and continuous hemodiafiltration, but infection spread into the retroperitoneal tissue and right thigh gradually. On ICU day 53, she died due to multiple organ failure.

Conclusions: Acute peritonitis due to spontaneous perforation of the CBD is very rare, and to our knowledge, this is the first report of biliary peritonitis in a patient receiving PD. Physicians should pay special attention to refractory peritonitis, including biliary peritonitis, occurring in patients receiving PD because some cases can be fatal.
\end{abstract}

Keywords: Peritoneal dialysis, Sepsis, Peritonitis, Spontaneous perforation, Common bile duct

\section{Background}

The number of patients receiving dialysis in Japan is over 300,000 , with $3 \%$ of them receiving peritoneal dialysis (PD). Dialysis patients are at a very high risk of death, and approximately $10 \%$ die per year, with no significant difference in mortality rate between hemodialysis and PD [1]. Among patients receiving dialysis, infection and cardiovascular disease are the two main causes of death, each accounting for approximately $25 \%$ of all mortality, while the former has been increasing in recent years [2]. While pneumonia explains $80 \%$ of mortality due to infection in the general population, pneumonia and sepsis each accounts for $40 \%$ of mortality due to infection in

\footnotetext{
* Correspondence: kamohara.hide@gmail.com

'Department of Intensive Care Medicine, Kumamoto University Hospital,

1-1-1 Honjo, Chuo-ku, Kumamoto 860-8556, Japan

Full list of author information is available at the end of the article
}

dialysis patients. Mortality due to sepsis is 30 times higher in patients receiving dialysis compared with that in the general population [3].

Bile duct injury is a rare, but potentially fatal, complication of endoscopic retrograde cholangiopancreatography or cholecystectomy. Although spontaneous perforation of the bile duct without iatrogenic or traumatic injury is very rare, it is critical to treat rapidly due to its high mortality [4].

\section{Case presentation}

A 73-year-old woman was admitted to our hospital for management of PD-related peritonitis. She had started PD for end-stage renal disease (ESRD) 6 months before, while the underlying disease remained unidentified. She was not previously diagnosed with diabetes or hypertension. Myeloperoxidase and proteinase-3 antineutrophil 
cytoplasmic antibody was negative, and she did not appear to exhibit vasculitis. She had undergone sigmoid colectomy and splenectomy previously as well as had a history of liver cirrhosis. The cause of liver cirrhosis was unknown. HBV and HCV were negative and her ChildPugh score was class B (8 points). The PD catheter was placed in the pouch of Douglas, but there were severe peritoneal adhesions due to her history of laparotomy. Urine volume was maintained at 1.0 to $1.5 \mathrm{~L} /$ day. In such a condition, the 2016 Kumamoto Earthquake forced her to stay in a car for evacuation, and PD was carried out there for weeks. Approximately 1 month after the earthquake, she presented with general malaise, hypotension, and turbidity of PD effluent. She was suspected to have PD-related peritonitis and was admitted to our hospital for management.

Upon hospitalization, her PD effluent was cloudy and contained $1200 / \mu \mathrm{L}$ white blood cells (WBCs) with $81 \%$ neutrophils, meeting the diagnostic criteria for PDrelated peritonitis [5]. Fever and abdominal pain were unremarkable at that time, and she was treated with intravenous meropenem. However, abdominal symptoms developed 2 days later, and treatment was switched to intraperitoneal vancomycin and gentamycin. Five days later, her PD effluent appeared bile-like, and infection extended to the retroperitoneum and abdominal cavity, as observed on computed tomography (CT). On the same day, emergent laparotomy was performed for biliary peritonitis. The cause of bile leakage was scrutinized but remained undetermined because of severe adhesion. The PD catheter was removed and drainage tubes were placed in the pouch of Douglas, at the dorsal side of the duodenum and retroperitoneum, and under the right part of the diaphragm. Endoscopic retrograde cholangiopancreatography was performed, which revealed perforation of the common bile duct (CBD) (Fig. 1). An endoscopic nasobiliary drainage tube was placed to reduce bile leakage. Despite the continuous drainage in the high care unit for several days, she developed cardiopulmonary arrest due to septic shock. After cardiopulmonary resuscitation and intubation, she was transferred to the intensive care unit (ICU).

Her level of consciousness was E1VTM1 using the Glasgow Coma Scale. Her vital signs were as follows: blood pressure, 101/68 $\mathrm{mmHg}$ under $0.08 \mu \mathrm{g} / \mathrm{kg} / \mathrm{min}$ noradrenaline; heart rate, $130 \mathrm{bpm}$; body temperature, $36.6{ }^{\circ} \mathrm{C}$; respiratory rate, 30 breaths/min; and $\mathrm{SpO}_{2}$, $100 \%$ under mechanical ventilation. Laboratory data indicated acute and sustained infection, including WBC count, $8300 / \mathrm{mm}^{2}$; C-reactive protein, $12.2 \mathrm{mg} / \mathrm{dL}$; total bilirubin, $2.7 \mathrm{mg} / \mathrm{dL}$; serum creatinine, $3.96 \mathrm{mg} / \mathrm{dL}$; arterial blood $\mathrm{pH}, 7.36$; and lactate, $3.01 \mathrm{mmol} / \mathrm{L}$ (Table 1). She did not present abdominal symptoms suggestive of acute pancreatitis or panperitonitis. Klebsiella

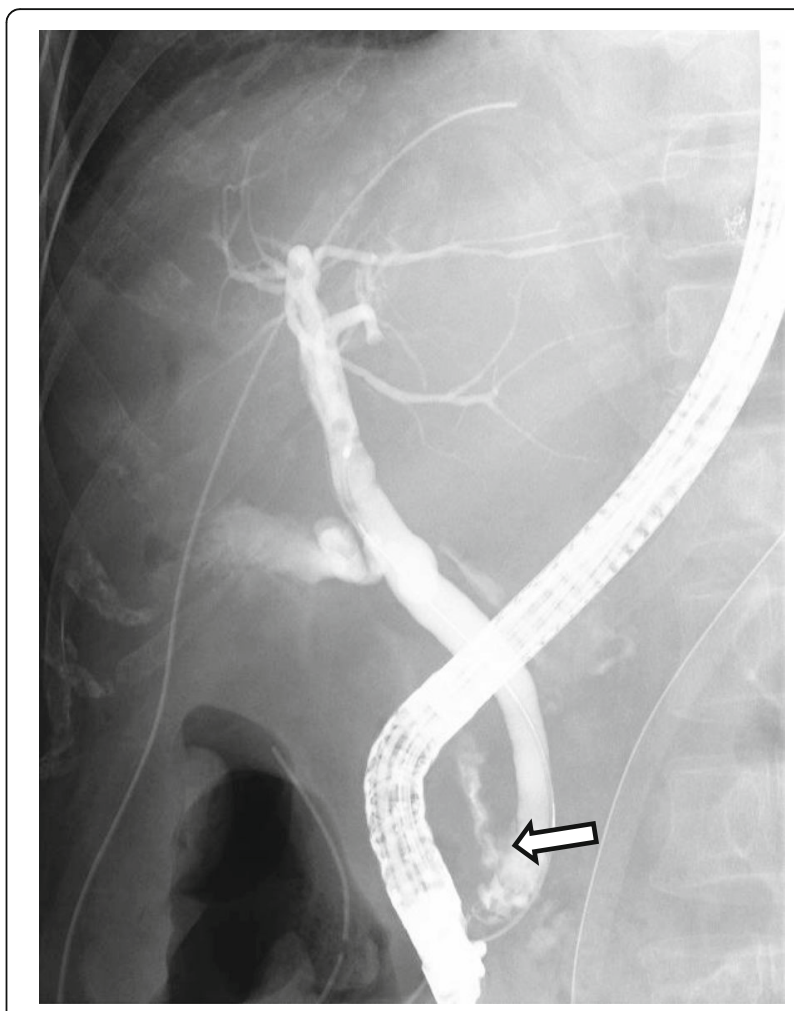

Fig. 1 Endoscopic retrograde cholangiopancreatography, revealing perforation of the common bile duct

pneumoniae was detected in the dialysis effluent, and tazobactam/piperacillin was administered as empiric therapy (Fig. 2). CT showed presence of free air in the peritoneal cavity, ascites, and mild edema of the gall bladder, but no stone was found in the gall bladder or CBD (Fig. 3). Bilateral pleural effusion with small nodules scattered in the lung field was also observed. Additional drainage tubes were placed in the retroperitoneum and around the perforation site, and intraperitoneal infection was managed by washing and drainage with continuous negative pressure. After nonresistant Escherichia coli, Candida albicans and Candida glabrata were detected from the ascites, and liposomal amphotericin B was added to the regimen. On ICU day 21, Mycobacterium tuberculosis was detected from the ascites, and she was administered antituberculosis agents, including isoniazid, rifampicin, ethambutol, and pyrazinamide. Chest CT showed persistent pleural effusion, atelectasis, ground-glass opacities, and scattered nodular lesions, suggesting that withdrawal from the ventilator would be difficult. Tracheotomy was performed on ICU day 29, and oxygenation was maintained under continuous positive airway pressure mode of the ventilator. She gradually became anuric and was supported by continuous hemodiafiltration. Despite such intensive therapies, infection with bile leakage spread 
Table 1 Clinical data of the patient on admission to the intensive care unit

\begin{tabular}{|c|c|c|c|}
\hline \multicolumn{2}{|c|}{ Blood biochemistry } & \multicolumn{2}{|c|}{ Blood count } \\
\hline $\mathrm{TP}(\mathrm{g} / \mathrm{dL})$ & 3.9 & WBC $(/ \mu \mathrm{L})$ & 8300 \\
\hline Alb $(g / d L)$ & 1.7 & Neut (\%) & 87.5 \\
\hline $\mathrm{Na}(\mathrm{mmol} / \mathrm{L})$ & 141 & Lymp (\%) & 7.1 \\
\hline $\mathrm{K}(\mathrm{mmol} / \mathrm{L})$ & 4.8 & $\mathrm{Hb}(\mathrm{g} / \mathrm{dL})$ & 10.1 \\
\hline $\mathrm{CL}(\mathrm{mmol} / \mathrm{L})$ & 112 & Plt $(\times 10 \wedge 4 / \mu \mathrm{L})$ & 2.4 \\
\hline $\mathrm{Ca}(\mathrm{mg} / \mathrm{dL})$ & 6.8 & & \\
\hline IP (mg/dL) & 5.7 & \multicolumn{2}{|c|}{ Coagulation } \\
\hline BUN (mg/dL) & 47.6 & PT-INR & 1.33 \\
\hline Cre (mg/dL) & 3.96 & APTT (\%) & 33 \\
\hline T-BiL (mg/dL) & 2.7 & $\mathrm{FDP}(\mu \mathrm{g} / \mathrm{ml})$ & 42.9 \\
\hline AST (U/L) & 54 & D-dimer $(\mu \mathrm{g} / \mathrm{mL})$ & 21.5 \\
\hline $\operatorname{ALT}(\mathrm{U} / \mathrm{L})$ & 9 & TAT $(\mathrm{ng} / \mathrm{mL})$ & 53.5 \\
\hline LD (U/L) & 636 & $\mathrm{PIC}(\mu \mathrm{g} / \mathrm{mL})$ & 0.8 \\
\hline үGTP (U/L) & 50 & & \\
\hline $\mathrm{ALP}(\mathrm{U} / \mathrm{L})$ & 350 & \multicolumn{2}{|c|}{ Arterial blood gas } \\
\hline Amy $(U / L)$ & 27 & \multicolumn{2}{|c|}{ (PSV; $\mathrm{FiO}_{2}$ 0.6, PEEP $10 \mathrm{mmHg}$, PS $15 \mathrm{mmHg}$ ) } \\
\hline CK (U/L) & 187 & $\mathrm{pH}$ & 7.36 \\
\hline Myo (ng/mL) & 2823 & $\mathrm{PaCO}_{2}(\mathrm{mmHg})$ & 29.1 \\
\hline CRP (mg/dL) & 12.2 & $\mathrm{PaO}_{2}(\mathrm{mmHg})$ & 94.3 \\
\hline PCT (ng/mL) & 9.3 & $\mathrm{HCO}_{3}-(\mathrm{mmol} / \mathrm{L})$ & 16.1 \\
\hline \multirow[t]{3}{*}{ BNP $(\mathrm{pg} / \mathrm{mL})$} & 5604 & $\mathrm{BE}(\mathrm{mmol} / \mathrm{L})$ & -8 \\
\hline & & Glu (mg/dL) & 181 \\
\hline & & Lac (mmol/L) & 3.01 \\
\hline
\end{tabular}

Abbreviations: Alb albumin, ALP alkaline phosphatase, $A L T$ alanin aminotransferase, Amy amylase, APTT activated partial thromboplastin time, $A S T$ aspartate aminotransferase, $B E$ base excess, $B N P$ brain natriuretic peptide, $B U N$ blood urine nitrogen, $C a$ calcium, $C K$ creatinine kinase, $C L$ chloride, $C r e$ creatinine, CRP C-reactive protein, $F D P$ fibrin degradation product, $\mathrm{FiO}_{2}$ fraction of inspired oxygen, $\gamma G T P$-glutamyltranspeptidase, Glu glucose, $\mathrm{Hb}$ hemoglobin, $\mathrm{HCO}_{3}$ bicarbonate, IP inorganic phosphorus, $\mathrm{K}$ potassium, LaC lactate, $L D$ lactate dehydrogenase, $L y m p$ lymphocytes, Myo myoglobin, $\mathrm{Na}$ sodium, Neut Neutrophil, $\mathrm{PaCO}_{2}$ partial pressure of carbon dioxide, $\mathrm{PaO}_{2}$ partial pressure of oxygen, PCT procalcitonin, PEEP positive end expiratory pressure, $P I C$ plasmin a2-plasmin inhibitor complex, Plt platelet, PS inspiratory pressure, PSV pressure support ventilation, $P T$-INR prothrombin time-international normalized ratio, TAT thrombin antithrombin complex, T-BiL total bilirubin, TP total protein, WBC white blood cell

into the peritoneal and retroperitoneal spaces, and new abscesses with hematomas formed and extended around the buttocks and right thigh. Her total bilirubin level reached $40 \mathrm{mg} / \mathrm{dL}$, and her sequential organ failure assessment score increased to 20. She died on ICU day 53 due to multiple organ failure.

\section{Mini-review of refractory peritonitis in patients receiving PD Etiology}

Peritonitis is a major contributing cause of death in patients receiving PD, accounting for 10 to $20 \%$ of cases. In recent years, technological improvements in PD connectology have decreased rates of PD-related peritonitis caused by Staphylococcus epidermidis and S. aureus. However, rates of PD-related peritonitis caused by gramnegative bacteria, polymicrobes, and fungi have remained steady [6]. Although PD-related peritonitis often is caused by peri- or intraluminal contamination with skin bacteria, it also can result from transvisceral or hematogenous spread of micro-organisms. Infection by gram-negative bacteria, polymicrobes, or fungi is associated with visceral disease. Kern et al. reported the odds ratio of a patient having visceral peritonitis as $66: 1$ in those with gram-negative bacteria or fungi in dialysate culture compared with those with gram-positive infection [7]. Influence of hypertonic fluid on peritoneal and intestinal microcirculation, presence of specific toxic substances in PD solution, and altered immune response induced by a chronic indwelling catheter may play pathophysiologic roles in development of intra-abdominal disorders [7]. In addition, age is reported to be a risk factor for refractory peritonitis.

\section{Diagnosis}

Patients with peritonitis usually present with cloudy PD effluent and abdominal pain. Effluent cell count including WBCs $>100 / \mu \mathrm{L}$ (after a dwell time of at least $2 \mathrm{~h}$ ) and $>$ $50 \%$ polymorphonuclear neutrophilic cells is highly suggestive of peritonitis [5]. In addition to peritoneal effluent culture, peripheral blood culture should be obtained if the patient is clinically septic, and antibiotic therapy should be initiated without waiting for culture results. After initiation of antibiotic therapy, usually, there is clinical improvement within 3 days. Refractory peritonitis is defined as failure of PD effluent to clear up after 5 days of appropriate antibiotic therapy. Presentation of sepsis or elevated dialysis effluent amylase level should increase the suspicion of serious intra-abdominal pathology [6].

\section{Management}

Further reductions in mortality due to PD-associated peritonitis are likely to depend on earlier diagnosis and better management of intra-abdominal pathology. Peritonitis that does not resolve after 3 days of appropriate antibiotic therapy or presentation of sepsis is more likely to represent continuous contamination requiring surgical intervention. Catheter removal is indicated in cases of refractory peritonitis. Prolonged attempts to treat refractory peritonitis by antibiotics without catheter removal are associated with extended hospital stay, peritoneal membrane damage, increased risk of fungal peritonitis, and excessive mortality [8]. Suspicion of visceral disease also should be high in patients infected by gram-negative bacteria, polymicrobes, or fungi and in those who fail to improve rapidly. 


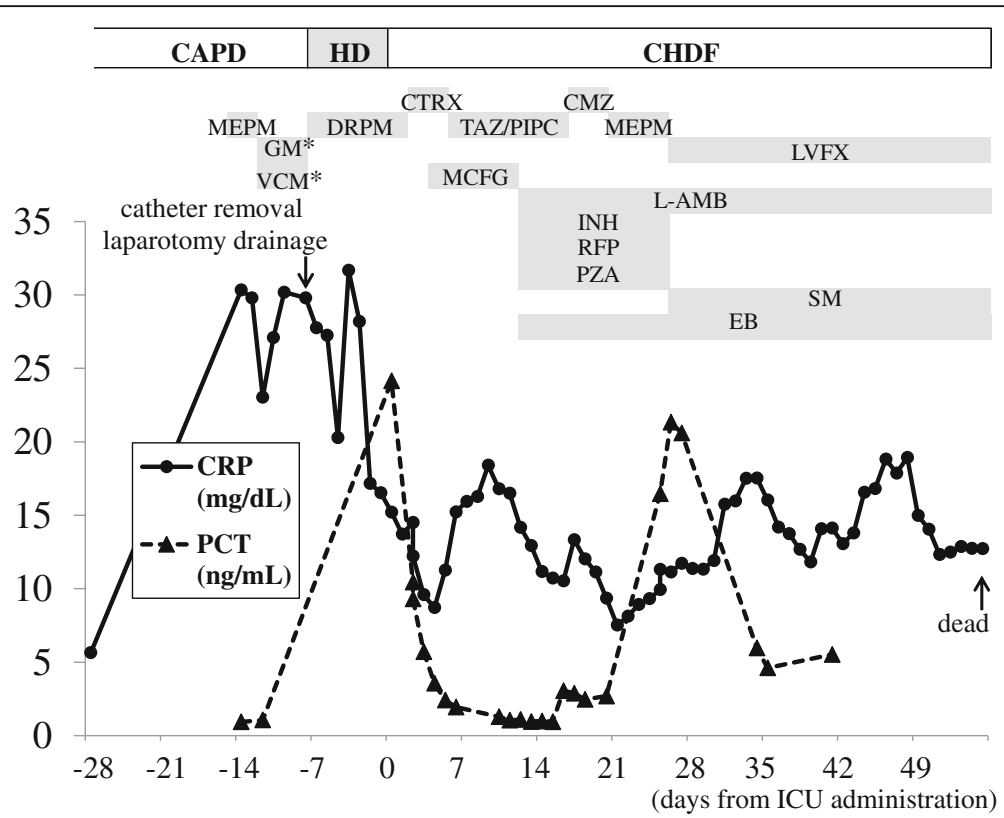

Fig. 2 Clinical course of the patient. Agents were administered intravenously, unless marked with an asterisk, which indicated intraperitoneal administration. Abbreviations: CAPD, continuous ambulatory peritoneal dialysis; CHDF, continuous hemodiafiltration; CMZ, cefmetazole; CTRX, ceftriaxone; DRPM, doripenem; EB, ethambutol; GM, gentamicin; HD, hemodialysis; INH, isoniazid; L-AMB, liposomal amphotericin B; LVFX, levofloxacin; MCFG, micafungin; MEPM, meropenem; PZA, pyrazinamide; RFP, rifampicin; SM, streptomycin; TAZ/PIPC, tazobactam/piperacillin; VCM, vancomycin
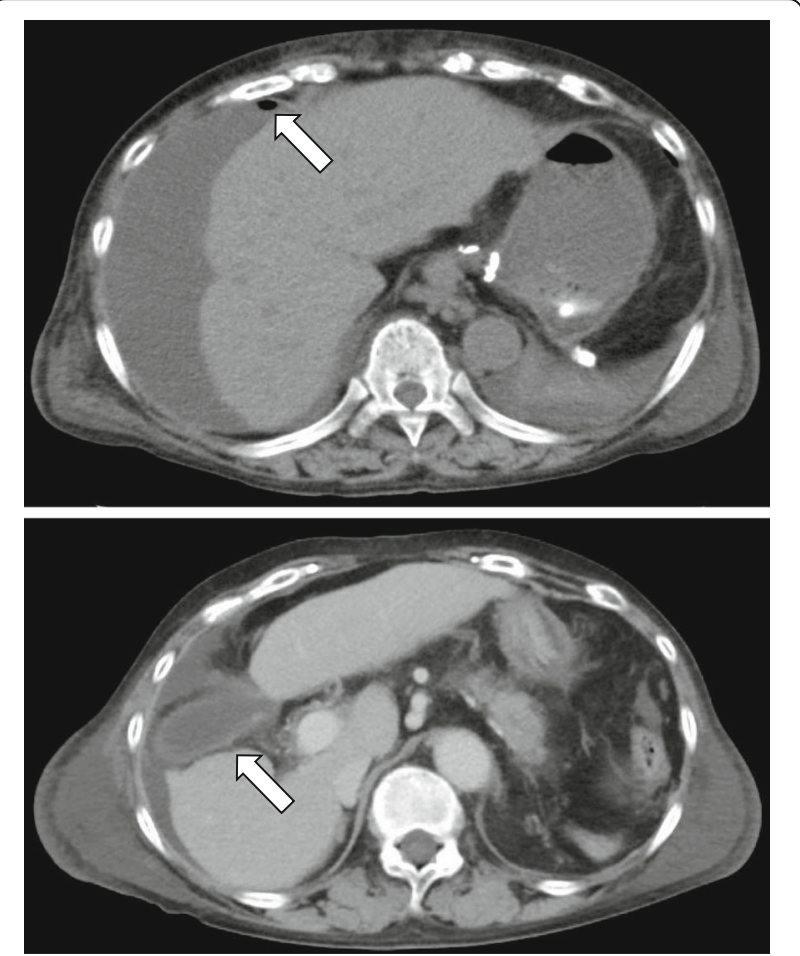

Fig. 3 Computed tomography upon ICU admission showed presence of free air in the peritoneal cavity, ascites, and mild edema of the gall bladder; however, no stones were found in the gall bladder or CBD

\section{Discussion}

Perforation peritonitis in patients receiving PD was first described in 1980 by Watson and colleagues [9]. Since then, there have been some reports of bowel perforation in patients receiving $\mathrm{PD}$, which occurred relatively late after catheter placement as an uncommon complication of PD [10]. Wang et al. summarized 28 cases of bowel perforation in patients receiving PD from 1980 to 2014 [11]. In their review, the colon was the most frequent site of perforation ( $64 \%$ of cases; sigmoid colon in $46 \%$ of cases) followed by the small intestine (25\%). There was no report of CBD perforation among them. Length of PD history varied from 0.5 to 54 months. Regarding primary disease of the kidney, chronic glomerulonephritis, nephrosclerosis, polycystic kidney disease, and diabetic nephropathy each accounted for 14\%; thus, there seems to be no kidney disease more likely to cause bowel perforation in the literature. The use of immunosuppressants and the presence of diverticulitis are recognized as risk factors for bowel perforation in patients receiving PD [11-13].

There are several reasons why diagnosis of peritonitis is sometimes difficult or delayed in patients receiving PD. First, the presence of free air or ascites may not become significant clues to diagnose intraperitoneal inflammation or gastrointestinal perforation. Second, peritoneal irritation symptoms may be alleviated during washing with the PD solution [11]. In the present case, the patient had a number of complications and 
comorbidities and suffered from an unexpected disaster. We think that, although difficult, the patient had to be consulted earlier to make an accurate diagnosis and begin treatment.

Spontaneous perforation of the extrahepatic bile duct without traumatic or iatrogenic injury is uncommon, but sometimes seen in children due to congenital weakness of the CBD or distal obstruction and pancreatic reflux [14]. Although nontraumatic spontaneous perforation by bile duct stones is rarely found in adults, it can cause fatal biliary peritonitis [4]. To our knowledge, the present case is the first report of perforation of the bile duct in a patient receiving PD, independent of catheterrelated mechanisms. Spontaneous perforation of the bile duct in adults may occur in the following situations: erosion by gallstones, increased intraductal pressure due to obstruction of the distal bile duct, thrombosis or ischemia of the bile duct wall, intramural infection of the duct as a result of cholangitis, regurgitation of pancreatic secretions into the bile duct, bile duct diverticulitis, and acute pancreatitis [15]. One of the most frequent causes of spontaneous perforation of the bile duct is biliary stones, especially after choledocholithiasis.

In our case, however, no gallstones, cysts, or diverticulums were observed. Occurrence of tuberculosis in this patient may suggest deteriorated cellular and humoral immunity under splenectomy. Although it is difficult to clarify the cause of spontaneous perforation of the CBD, considering the fact that dialysis patients are largely immune compromised [16], we suppose that persistent peritonitis would have extended to the bile duct or caused circulatory failure locally. We speculated that the patient's compromised immune function in addition to ESRD, tuberculosis, liver cirrhosis, elderly age, splenectomy, and frequent abdominal surgery history caused peritonitis comprehensively and Klebsiella pneumoniae spread to the abdominal cavity by bacterial translocation. Laparotomy indicated the severity of inflammation located in the retroperitoneum cavity below the liver. While there was edema, there was no gangrenous wall change or increased thickness in the gall bladder. ERCP demonstrated no significant evidence of inflammation, such as a stone, dilatation, or stenosis in the bile duct and gallbladder after operation. Although gallbladder edema was also observed in $\mathrm{CT}$, there was no mucosal ischemic finding, inflammation spreading to the surrounding of serous membrane, or bile duct extension. Additionally, our patient did not exhibit right hypochondrium pain nor any increase in biliary enzyme level. Therefore, we reasoned that the patient likely did not have primary cholecystitis or cholangitis. We instead considered that the perforation of the bile duct could be caused by local secondary inflammation. The control of bile leakage and, therefore, biliary peritonitis was severely complicated. Finally, inflammation and abscess formation spread toward the retroperitoneum and around the buttocks and thigh, along with development of tuberculous and fungal peritonitis. Such comorbid disorders together contributed to this patient's death. Early diagnosis and complete control of bile leakage are critical to rescuing elderly patients with ESRD and biliary peritonitis.

\section{Conclusions}

Here, we report the first case of refractory peritonitis by spontaneous perforation of the CBD in a patient receiving PD. Physicians should be particularly attentive to refractory peritonitis, including biliary peritonitis, occurring in patients receiving PD since some cases can be fatal.

\section{Abbreviations \\ CBD: Common bile duct; CT: Computed tomography; ICU: Intensive care unit; PD: Peritoneal dialysis; WBC: White blood cell}

\section{Acknowledgements}

Not applicable.

Funding

None.

Availability of data and materials

The datasets generated during and/or analyzed during the current study are available from the corresponding author upon reasonable request.

Authors' contributions

$\mathrm{MH}, J Y, K T, M E, K S, S N, D H, H B, K M, Y K, T M$, and HK provided discussion and treatment of the patient. $\mathrm{MH}$ collected the data and wrote the manuscript. $\mathrm{MH}, \mathrm{MM}$, and $\mathrm{HK}$ reviewed and revised the manuscript. All authors read and approved the final manuscript.

Ethics approval and consent to participate

Not applicable.

\section{Consent for publication}

Written informed consent was obtained in advance from the patient's family for the publication of this case report and any accompanying images.

\section{Competing interests}

The authors declare that they have no competing interests.

\section{Publisher's Note}

Springer Nature remains neutral with regard to jurisdictional claims in published maps and institutional affiliations.

\section{Author details \\ ${ }^{1}$ Department of Intensive Care Medicine, Kumamoto University Hospital, 1-1-1 Honjo, Chuo-ku, Kumamoto 860-8556, Japan. ${ }^{2}$ Department of Nephrology, Kumamoto University Graduate School of Medical Sciences, Kumamoto, Japan. ${ }^{3}$ Department of Gastroenterological Surgery, Kumamoto University Graduate School of Medical Sciences, Kumamoto, Japan.}

Received: 22 May 2017 Accepted: 20 September 2017 Published online: 30 November 2017

\section{References}

1. Suzuki K, Konta T, Ichikawa K, Ikeda A, Niino H, Hoshikawa M, et al. Comparison of mortality between Japanese peritoneal dialysis and hemodialysis patients: a 5-year multicenter follow-up study. Int J Nephrol. 2012;2012:231018 
2. Masakane I, Nakai S, Ogata S, Kimata N, Hanafusa N, Hamano T, et al. An overview of regular dialysis treatment in Japan (as of 31 December 2013). Ther Apher Dial. 2015;19:540-74.

3. Wakasugi M, Kawamura K, Yamamoto S, Kazama JJ, Narita I. High mortality rate of infectious diseases in dialysis patients: a comparison with the general population in Japan. Ther Apher Dial. 2012;16:226-31.

4. Kang SB, Han HS, Min SK, Lee HK. Nontraumatic perforation of the bile duct in adults. Arch Surg. 2004;139:1083-7.

5. Li PK, Szeto CC, Piraino B, de Arteaga J, Fan S, Figueiredo AE, et al. ISPD peritonitis recommendations: 2016 update on prevention and treatment. Perit Dial Int. 2016;36:481-508.

6. Faber MD, Yee J. Diagnosis and management of enteric disease and abdominal catastrophe in peritoneal dialysis patients with peritonitis. Adv Chronic Kidney Dis. 2006;13:271-9.

7. Kern EO, Newman LN, Cacho CP, Schulak JA, Weiss MF. Abdominal catastrophe revisited: the risk and outcome of enteric peritoneal contamination. Perit Dial Int. 2002;22:323-34.

8. Choi P, Nemati E, Banerjee A, Preston E, Levy J, Brown E. Peritoneal dialysis catheter removal for acute peritonitis: a retrospective analysis of factors associated with catheter removal and prolonged postoperative hospitalization. Am J Kidney Dis. 2004;43:103-11.

9. Watson $L C$, Thompson JC. Erosion of the colon by a long-dwelling peritoneal dialysis catheter. JAMA. 1980;243:2156-7.

10. Ratajczak A, Lange-Ratajczak M, Bobkiewicz A, Studniarek A. Surgical management of complications with peritoneal dialysis. Semin Dial. 2017;30:63-8.

11. Wang R, Chen Z, Wang J, Zhang X, Shou Z, Chen J. Delayed bowel perforation in a peritoneal dialysis patient: a case report and literature review. Perit Dial Int. 2014;34:460-6.

12. Nakamura H, Kitazawa K, Kato K, Inada Y, Kato N, Takahashi M, et al. Stercoral perforation of the sigmoid colon in a patient undergoing CAPD: case report. Perit Dial Int. 2004;24:399-401.

13. Imai $\mathrm{H}$, Satoh K. Perforated diverticulitis after barium enema examination in a patient on CAPD. Nephrol Dial Transplant. 1997;12:2758-60.

14. Krishna RP, Lal R, Sikora SS, Yachha SK, Pal L. Unusual causes of extrahepatic biliary obstruction in children: a case series with review of literature. Pediatr Surg Int. 2008;24:183-90.

15. Ishii K, Matsuo K, Seki H, Yasui N, Sakata M, Shimada A, et al. Retroperitoneal biloma due to spontaneous perforation of the left hepatic duct. Am J Case Rep. 2016;17:264-7.

16. Betjes MG. Immune cell dysfunction and inflammation in end-stage renal disease. Nat Rev Nephrol. 2013;9:255-65.

\section{Submit your next manuscript to BioMed Central and we will help you at every step:}

- We accept pre-submission inquiries

- Our selector tool helps you to find the most relevant journal

- We provide round the clock customer support

- Convenient online submission

- Thorough peer review

- Inclusion in PubMed and all major indexing services

- Maximum visibility for your research

Submit your manuscript at www.biomedcentral.com/submit

) Biomed Central 